CASE REPORT

\title{
Lymph node tuberculosis - The ENT surgeon approach in four cases
}

\author{
Daniela Vrinceanu', Mihai Dumitru', Maria Sajin², Carmen Maria Salavastru³, Adrian Costache² \\ ${ }^{1}$ ENT Department, University Emergency Hospital, Bucharest, Romania \\ ${ }^{2}$ Department of Pathology, "Carol Davila" University of Medicine and Pharmacy, Bucharest, Romania \\ ${ }^{3}$ Department of Dermato-Venerology, "Carol Davila” University of Medicine and Pharmacy, Bucharest, Romania
}

\section{ABSTRACT}

BACKGROUND. Lymph node tuberculosis is a pathology with an increasing incidence and prevalence in middle income countries. MATERIAL AND METHODS. We present a series of 4 cases with cervical lymph node tuberculosis. We review current principles of diagnosis and treatment from the perspective of the ENT surgeon in a tertiary university clinic.

RESULTS. In each case we underline diagnosis difficulties and treatment options. These cases presented management difficulties due to associated morbidities. All cases underwent surgical excision of the afflicted lymph nodes with subsequent microscopic confirmation of tuberculosis. We illustrate key concepts leading to the microscopy diagnosis of lymph node tuberculosis.

CONCLUSION. There are various surgical incidents and accidents that the young surgeon must be aware of when approaching neck tuberculous lymph nodes. Further referral of the patient for long-term tuberculosis treatment is mandatory. All patients were supervised for a minimum of 1 year after the initial diagnosis and treatment with no sign of recurrence. A close cooperation between the ENT surgeon, the infectious disease specialist and the pathologist is the key to an optimum approach to lymph node tuberculosis at the head and neck level.

KEYWORDS: neuroendocrine carcinoma, nasopharyngeal carcinoma, second primary malignancy, small cell carcinoma.

\section{INTRODUCTION}

Extrapulmonary tuberculosis accounts for 15$30 \%$ of the total cases with tuberculosis, and the lymph node affliction is one of the most frequent ${ }^{1}$. From all peripheral lymph nodes, $70-90 \%$ are at the neck level and rarely in the inguinal, axillary, epitrochlear areas ${ }^{2}$. At the neck level, the most affected nodules are jugular, submandibular, spinal, or at the parotid level. Actually, lymph node tuberculosis can be encountered in any neck region and it is bilateral in $20 \%$ of the cases ${ }^{1,2}$. The search for an active pulmonary lesion is positive in immunocompromised patients ${ }^{3}$. Given the fact that in Romania tuberculosis is prevented through a nationwide vaccination programme, most cases are found in poor areas and risk population ${ }^{4}$. The clinical aspect varies from neck lymph nodes with an initial tough consistency to multiple lymph nodes uniting into a hard mass adherent to the skin and the surrounding tissues, leading to ulcerations and scaring $^{5}$. During surgical ablation, the lymph nodes can present necrosis and it is necessary to gather tissue for a bacteriology exam, but the gold standard for diagnosis is the pathology result ${ }^{6}$.

\section{MATERIAL AND METHODS}

We will present a series of 4 cases with surgically removed neck lymph nodes and the pathology confirmed as lymph node tuberculosis. These cases were admitted to the ENT Department of the Bucharest University Emergency Hospital. All patients were females, aged 54, 37, 64 and 78 respectively. The first three presented with neck lymph nodes in

Corresponding author: Mihai Dumitru, MD, PhD, ENT Specialist, Anatomy Department, University Emergency Hospital, "Carol Davila" University of Medicine and Pharmacy, Bucharest, Romania, 1 Sarandy Frosa, Bl. 33, Sc. A, Ap. 12, District 1, 011231, Bucharest, Romania 


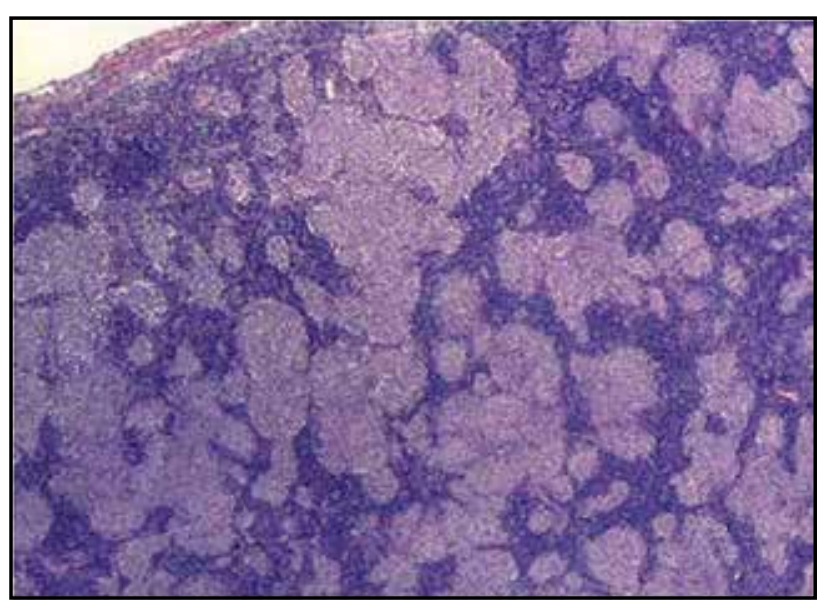

Figure 1 Confluent Koster follicles - microscopic aspect specific to tuberculosis. Differential diagnosis with Besnier-Boeck-Schaumann disease. (Hematoxylin-Eosin, 100x magnification)

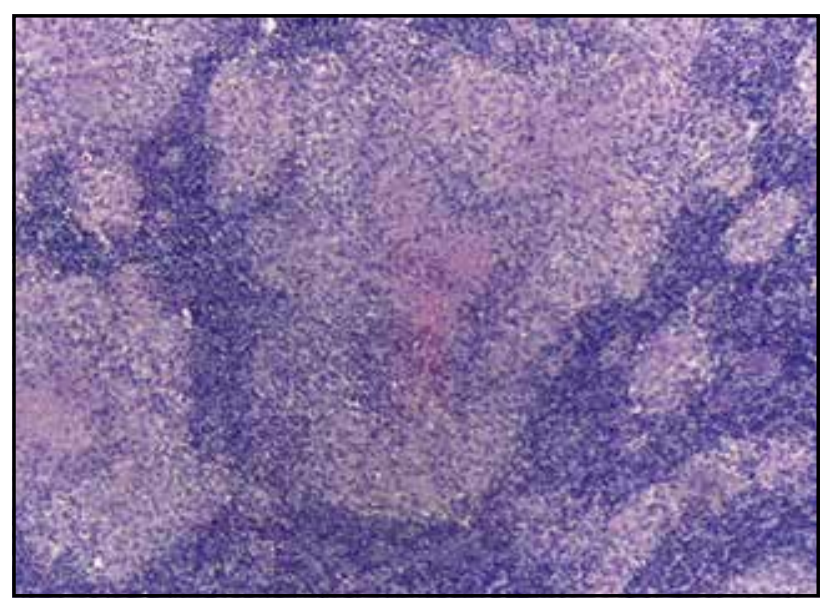

Figure 2 Detail of Figure 1 with Koster epithelioid follicles with central caseous necrosis and without Langhans cells specific to a low immunity response. (Hematoxylin-Eosin, 100x magnification)

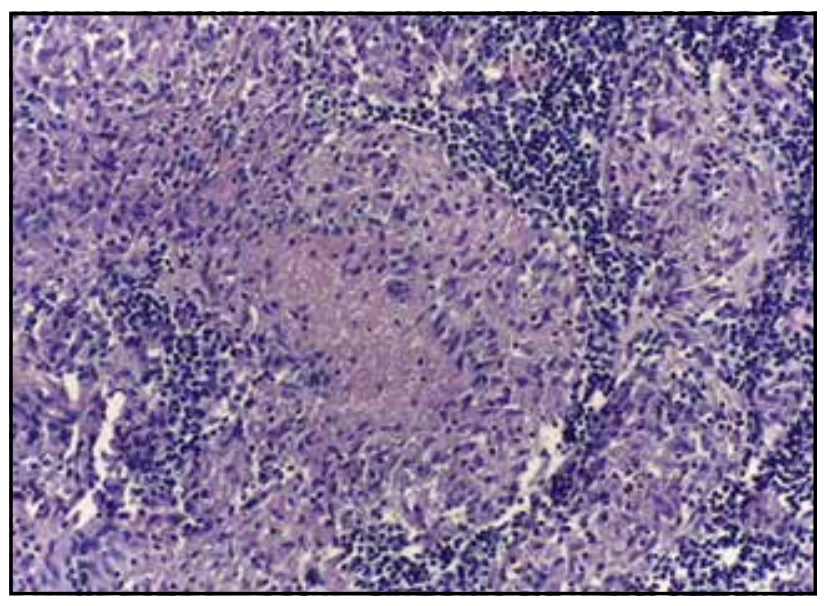

Figure 3 Detail of Figure 2 at higher magnification. (HematoxylinEosin, 200x magnification) progressive growth during the previous 6 months. The fourth patient presented an anterior tracheal lymph node with a quick progression in the previous 3 months and a fistula through the skin 24 hours before admittance.

The clinical exam and imaging raised the suspicion of lymphatic proliferative syndrome with indication for biopsy. The surgical procedures were performed under general anaesthesia. Given the pathology results, the patients were referred to the National Institute of Pneumology for a 6 to 9 -month course of treatment. Patients were followed up for 12 months, with complete recovery.

\section{RESULTS}

\section{Case 1}

Blood tests without inflammatory syndrome, normal chest radiography and abdominal sonography without lymph nodes and normal spleen and liver. The neck ultrasound revealed: a lymph node at the level of the parotid gland, well delineated, hypoechoic, with dimensions of $21 / 15 / 27 \mathrm{~mm}$; posterior to the sternocleidomastoid muscle, another lymph node of $11 / 5 \mathrm{~mm}$ and, on the left side, multiple lymph nodes reactive in nature under $10 \mathrm{~mm}$.

During surgery, we removed two lymph nodes of 3 and $2.5 \mathrm{~cm}$ respectively. The pathology result described epithelial granulomas with central necrosis characteristic for lymph node tuberculosis (Figure 1, Figure 2, Figure 3).

The patient had a positive evolution under a 6-month specialist treatment regimen at "Marius Nasta" National Pneumology Institute.

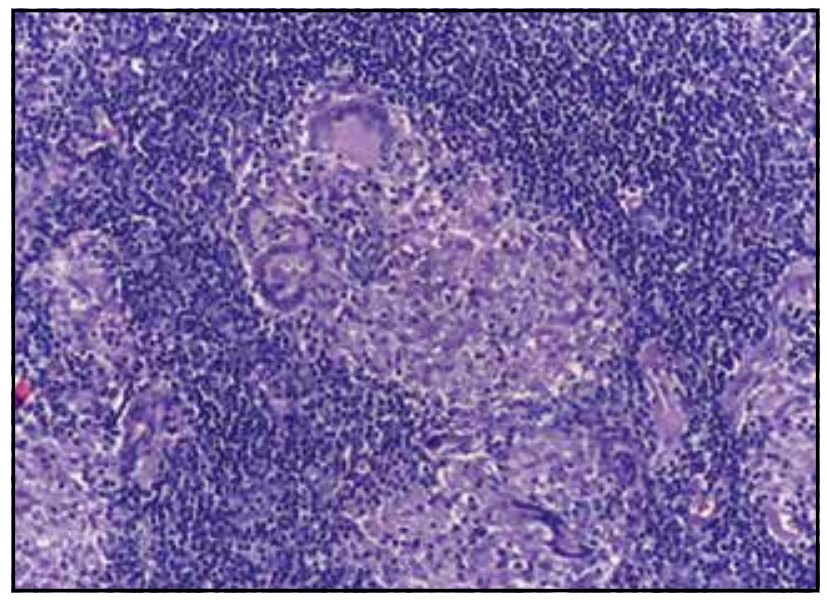

Figure 4 Tuberculosis with gigantic Koster follicles: Langhans cells (upper left corner of the central Koster follicle and in the lower part of the follicle) are specific but not compulsory diagnosis elements and show a good host immunity response.(Hematoxylin-Eosin, 200x magnification) 


\section{Case 2}

Young patient admitted with multiple right neck lymph nodes in evolution for 6 months. Blood tests excluded an inflammatory syndrome, HIV and hepatitis.

The neck ultrasound showed multiple lymph nodes ranging between 10 to $20 \mathrm{~mm}$ in length, neighbouring the major vascular bundle. At submandibular level, there was another lymph node of $46 / 18 \mathrm{~mm}$ with thickened cortex, hypoechoic and the hilum reduced to a central hyperechoic band. The vascular Doppler signal was increased at the level of the lymph node. On the left side, the lymph nodes had an inflammatory aspect.

The abdominal ultrasound was negative for lymph nodes, spleen or liver involvement.

The surgical removal of the right submandibular mass produced a lymph node with high consistency, of 20/16/12mm, and various white areas on

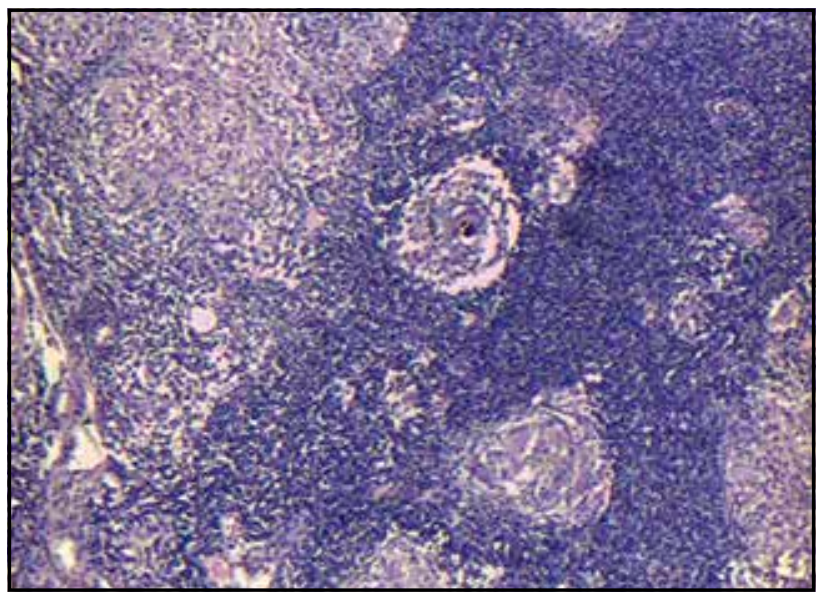

Figure 5 Tuberculosis with Koster follicles, mainly epithelioid, with rare Langhans cells (central) given a low immunity response. (Hematoxylin-Eosin, 100x magnification)

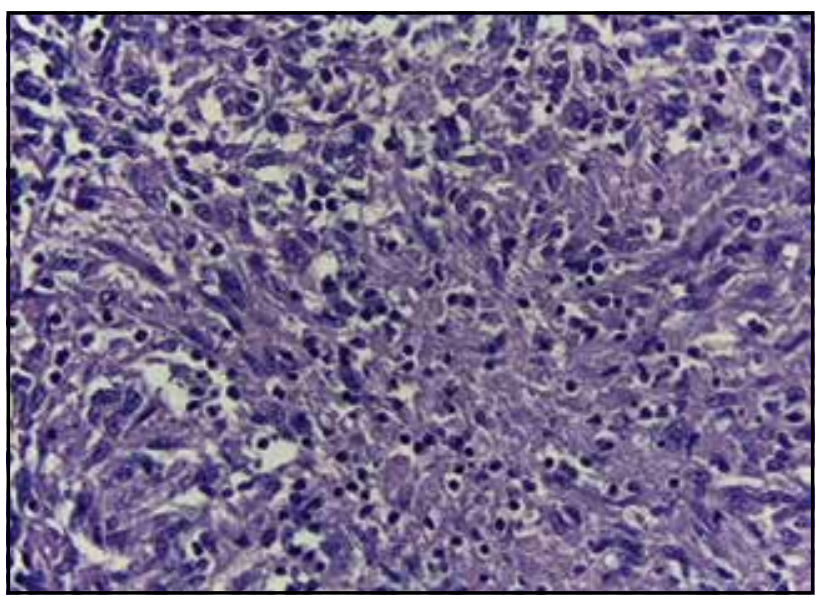

Figure 7 Detail from Figure 6 with epithelioid cells and lymphocytes. (Hematoxylin-Eosin, 400x magnification) the central section. The pathology result underlines lymph node tuberculosis with areas of necrosis through calcification (Figure 4).

The patient fully recovered under a 6-month tuberculostatic regimen.

Specific to this case is the fact that the patient has been previously exposed to the pulmonary tuberculosis in her father for one year.

\section{Case 3}

The patient aged 54 with chronic hepatitis C under interferon treatment and a history of pulmonary tuberculosis (TB) 2 years before presented with a left neck lymph node with progressive growth during the previous 6 months.

Blood tests were within the normal range given the patient's age. The Chest x-ray showed TB scarring.

The neck ultrasound recorded necrotizing lymph nodes. The CT-scan of the neck and thorax

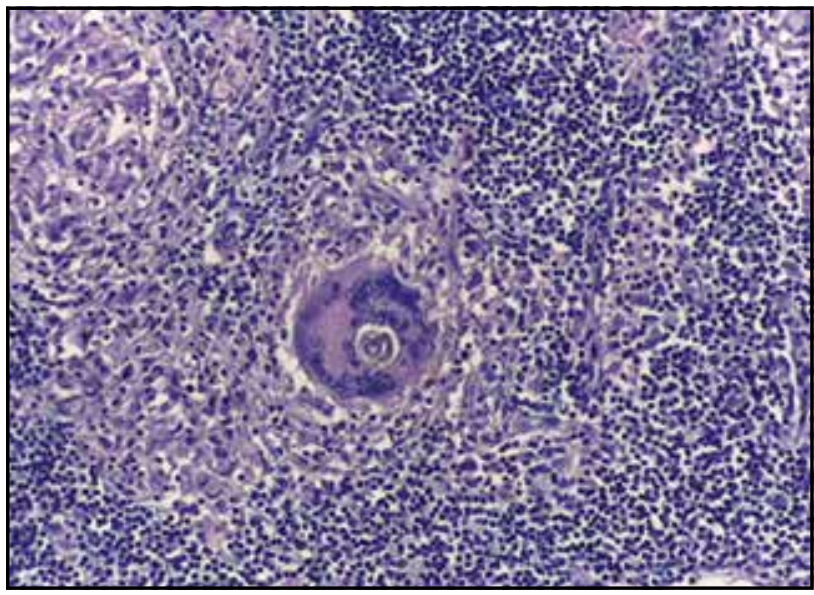

Figure 6 Detail from Figure 5 with a Langhans cell (in the center of the Koster follicle), epithelioid cells and lymphocytes. (HematoxylinEosin, 200x magnification)

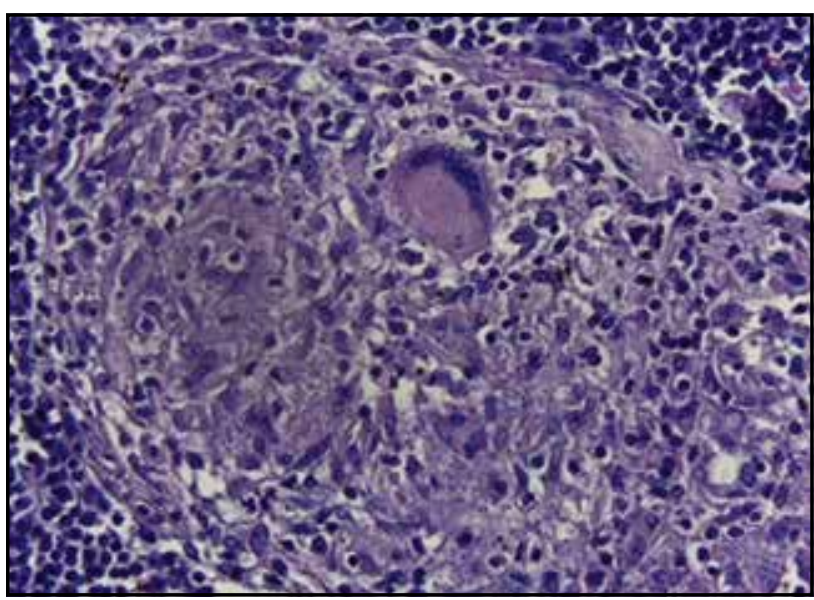

Figure 8 Detail from Figure 6 with a Langhans Cell, epithelioid cells and lymphocytes. (Hematoxylin-Eosin, 400x magnification) 


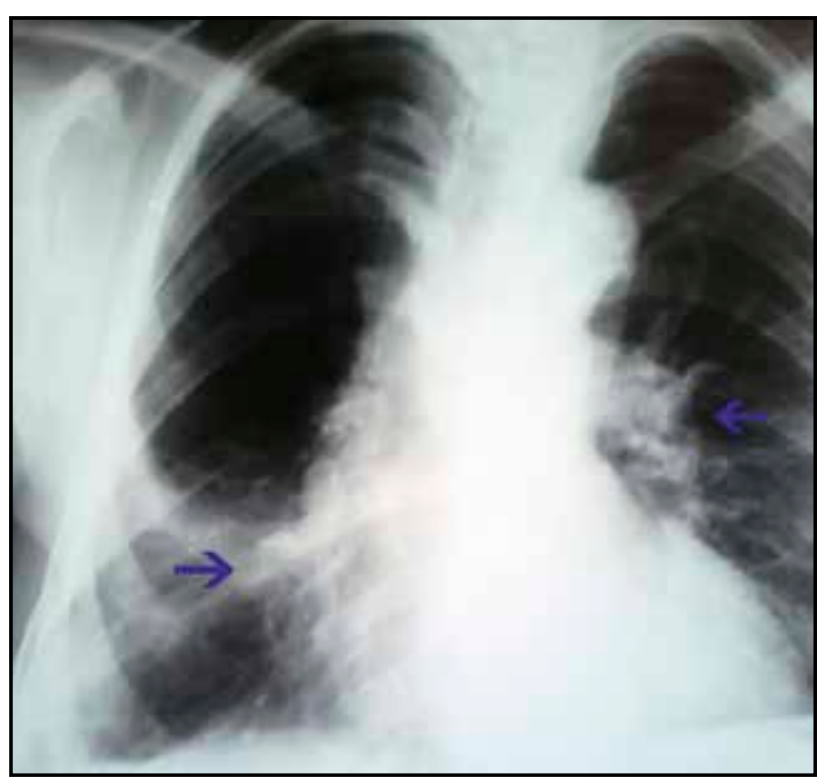

Figure 9 Chest X-ray with bilaterally enlarged hilum (arrows).

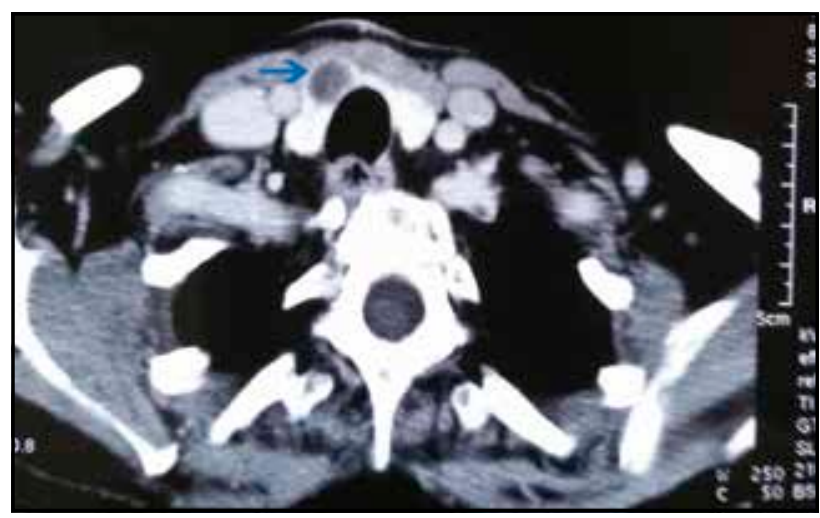

Figure 10 Neck CT-scan, axial slice - the arrow marks the thyroid lesion.

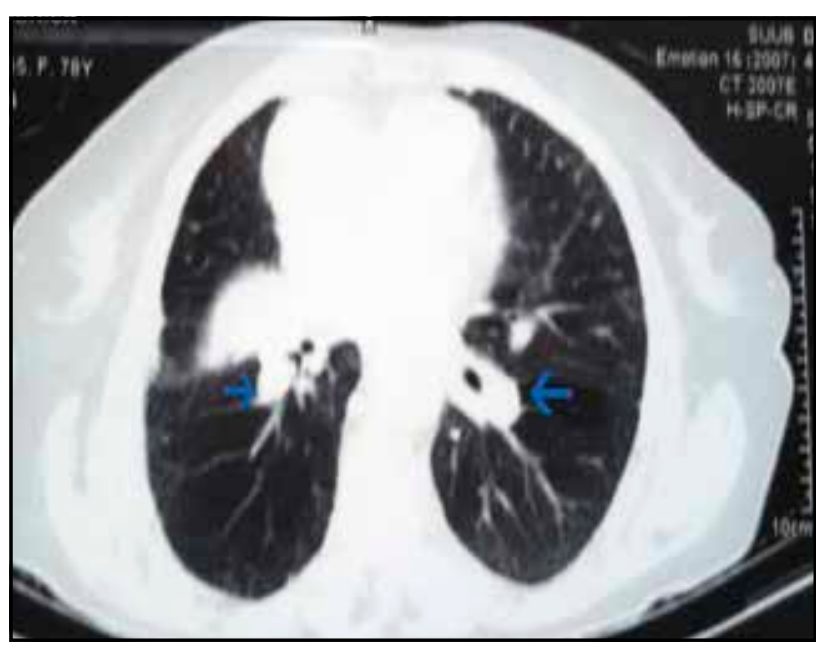

Figure 11 Chest CT-scan, axial slice - the arrows mark the expanding lesions. described a mass with mixed fluid content, of $2.7 / 2.3 \mathrm{~cm}$ axially and $3 \mathrm{~cm}$ longitudinally, on the left side of the neck, posterior to the submandibular gland and lateral to the main vascular bundle, but with an initial diagnosis of a brachial cyst.

The surgical excision revealed a cystic lymph node, with central necrosis, adherent to the internal jugular vein. The pathologic exam described an outer surface with irregularities and dimensions of 22/14/14 mm. The microscopic view contained Langhans gigantic cells in a specific chronic granuloma reaction (Figures 5, 6, 7 and 8).

The patient underwent specific medical treatment for 9 months with complete recovery.

\section{Case 4}

This female patient, aged 78 , had a previous history of thyroid goiter, with later development of 2 neck masses within a 3-month time span, with subsequent skin burst in 24 hours. The pulmonary plain X-ray presented a bilaterally enlarged hilum with multiple lesions, Figure 9.

The neck CT-scan described an oval-shaped structure, with inferior contact with the thyroid isthmus, with a thick wall gaining contrast media and fluid content, with overall dimensions of $23 / 20 \mathrm{~mm}$ and neighbouring a skin thickening of up to $5 \mathrm{~mm}$ along with bilateral superior jugular lymph nodes (Figure 10). The thorax CT-scan with IV contrast described a central expanding process at the level of the pulmonary hilum and a central necrosis with an overall dimension of $20 / 19 \mathrm{~mm}$, with other 4 lymph nodes at the pulmonary level and nodular structures in the pulmonary parenchyma (Figure 11).

The microscopic exam revealed puss with Acinetobacter baumannii, but it was negative for tuberculosis.

Under local anaesthesia, given the advanced age of the patient, we identified a cystic mass in front of the trachea, below the thyroid, adherent to the adjacent structures and requiring resection of the superficial skin and fistula. The pathology result confirmed a granulomatous inflammation without certainty of tuberculosis (Figure 12). Further excision of the axillary nodes by the thoracic surgeon confirmed lymph node tuberculosis. The patient was subjected to specific medical therapy. Unfortunately, the patient subsequently died due to multiple organ system failure.

\section{DISCUSSIONS}

Lab exams are mildly altered with lymphocytosis. Serology exams for Epstein-Barr virus, cytomegalovirus, Toxoplasma Gondii, Toxocara Canis, HIV 


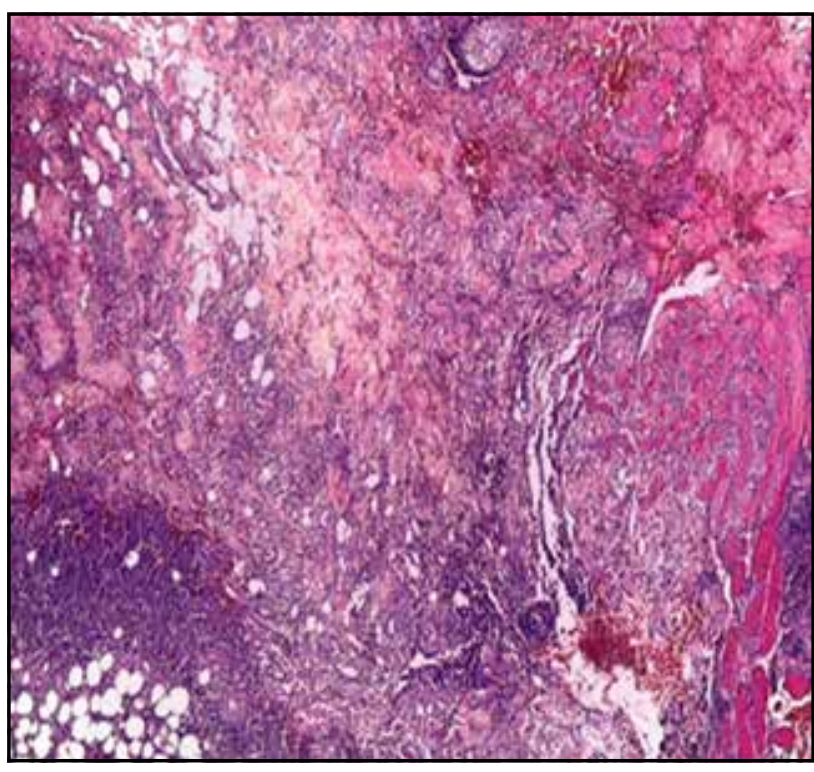

Figure 12 Wide field image: lymph nodes neighbouring muscle fibers (centre and lower right), reactive in nature with a Koster follicle on the left. (Hematoxylin-Eosin, 40x magnification)

and Treponema Pallidum is mandatory ${ }^{7}$. The chest $\mathrm{x}$-ray presents tuberculosis sequelae. The aspiration cytology presents epithelioid cells and positive cultures for Loffler bacillus ${ }^{8}$.

In our department, we prefer direct excision of the lymph node instead of aspiration cytology, because it takes 8 weeks of incubation at $37^{\circ} \mathrm{C}$ to have a positive isolation of mycobacteria on culture.

In cases with granuloma lymph nodes without necrosis, we order a supplementary immunology exam with $\mathrm{PCR}^{9}$.

The ENT clinical exam must exclude other types of tumors. The imaging exam offers details for the surgical approach and it is also useful for the healing comparison for the following 6 months to 1 year. The differential diagnosis includes malignant lymphoma, sarcoidosis or metastasis from other neck tumors ${ }^{10}$.

The immunity status is very important because the first 2 cases had poor healthcare and the third presented also infection with the hepatitis $\mathrm{C}$ virus. In the last case, the evolution towards exitus derived from lack of the immunity response and associated pathology.

\section{CONCLUSIONS}

We present 3 cases of isolated tuberculous lymph node involvement and 1 case with both neck and mediastinum tuberculous lymph nodes. Imaging using ultrasound and neck CT supports the clinical diagnosis. The surgical excision is the only viable method for obtaining pathology material. The pathology exam is the gold standard for diagnostic certainty. The medical treatment for tuberculosis spans over a period of 6 to 9 months, with multiple checks till clinical and serology healing.

Conflict of interest: The authors have no conflict of interest.

Contribution of authors: All authors have equally contributed to this work.

\section{REFERENCES}

1. Benjelloun A, Darouassi Y, Zakaria Y, Bouchentouf R, Errami N. Lymph nodes tuberculosis: a retrospective study on clinical and therapeutic features. Pan Afr Med J. 2015;20:65. DOI: 10.11604/ pamj.2015.20.65.5782. eCollection 2015.

2. Ryu YJ. Diagnosis of pulmonary tuberculosis: recent advances and diagnostic algorithms. Tuberc Respir Dis (Seoul). 2015;78(2):64-71. DOI 10.4046/trd.2015.78.2.64.

3. Martinez BG, Di Martino OB, Rodriguez MM, Knopfelmacher O, Bolla de Lezcano L. Ganglionar tuberculosis with skin involvement (scrofuloderma) in an inmmunocompetent patient. A case report. N Dermatol Online. 2011;2(3):130-4.

4. Branco C, Subtil J, Tomas L, Pereira AM. Tuberculose ganglionar cervical: a propósito de um caso clínico. Tuberculosis lymphadenitis: case report. Rev Port ORL. 2005;43(3):257-64.

5. Costache A, Dumitru M, Anghel I, Cergan R, Anghel AG, Sarafoleanu C. Ultrasonographic anatomy of head and neck - a pictorial for the ENT specialist. Med Ultrason. 2015;17(1):104-8. DOI : 10.11152/ mu.2013.2066.717.aco.

6. Deveci HS, Kule M, Kule ZA, Habesoglu TE. Diagnostic challenges in cervical tuberculous lymphadenitis: A review. North Clin Istanb. 2016;3(2):150-5. DOI: 10.14744/nci.2016.20982.

7. Zaldivar RFR, Hurtado LLM, Ramirez TD. Tuberculous lymphadenopathy of the neck: A diagnosis that should not be forgotten. Cir Gen. 2004;26(3):177-80.

8. Kumar H, Chandanwale SS, Gore CR, Buch AC, Satav VH, Pagaro PM. Role of fine needle aspiration cytology in assessment of cervical lymphadenopathy. Med J DY Patil Univ. 2013;6:400-4. DOI: 10.4103/09752870.118287.

9. Baek CH, Kim SI, Ko YH, Chu KC. Polymerase chain reaction detection of Mycobacterium tuberculosis from fine-needle aspirate for the diag-

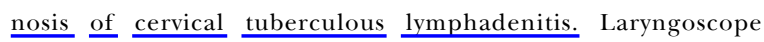
2000;110(1):30-4.

10. Kanlikama M, Gokalp A. Management of mycobacterial cervical lymphadenitis. World J Surg. 1997;21 (5):516-9. 